\title{
Finite groups with many metacyclic subgroups
}

\author{
Adolfo BALlester-Bolinches AND JOHN COSSEY
}

\begin{abstract}
The aim of this paper is to characterise the finite non-nilpotent groups in which every 2-generator subgroup is metacyclic.

Mathematics Subject Classification (2010): 20D10 (primary); 20D20 (secondary).
\end{abstract}

\section{Introduction}

The recovery of information about the structure of a finite group from information about its subgroups has a long history. Here we will be concerned with the influence of 2-generator subgroups on the structure of a finite group. If a group has 2-generator subgroups in a class $\mathcal{X}$ which is subgroup-closed and minimal non$\mathcal{X}$-groups are 2-generator then the group is in $\mathcal{X}$. This is true for the classes of soluble [8, 14], supersoluble [6] and nilpotent [9, Satz III.5.2] groups. Minimal non-metacyclic $p$-groups ( $p$ a prime) have been classified by Blackburn [2, Theorem 3.2]. These groups are all 3-generator and so the class of groups with all 2generator subgroups metacyclic contains non-metacyclic groups. For convenience, we denote the class of finite groups with 2-generator subgroups metacyclic by $\mathcal{M}$. Note that $\mathcal{M}$ is a subgroup and quotient closed class.

For odd primes $p$, the classification of $p$-groups in $\mathcal{M}$ is easy. If $G \in \mathcal{M}$, then $G$ can not contain a non-abelian section of order $p^{3}$ and exponent $p$, since such a section is not metacyclic. It then follows by [13, Lemma 2.3.3] that $G$ is a modular group. Conversely it follows from [13, Theorem 2.3.1] that a modular p-group has all 2-generator subgroups metacyclic. Mann [12] showed that the class of monotone 2-groups coincides with the class of 2-groups in $\mathcal{M}$. These groups have been classified by Crestani and Menegazzo [3] and we refer the reader to that paper for details.

Received January 24, 2014; accepted in revised form July 14, 2014.

Published online February 2016. 
Our aim here is to complete the classification of finite groups in $\mathcal{M}$ by classifying all the non-nilpotent finite groups in this class.

If $p$ is a prime, we denote a Sylow $p$-subgroup of a group $G$ by $G_{p}$ and if $\pi$ is a set of primes we denote a Hall $\pi$-subgroup by $G_{\pi}$.

Theorem 1.1. A non-nilpotent group $G$ has 2-generator subgroups metacyclic if and only if

(i) $G$ is supersoluble and metabelian, with Sylow subgroups modular for odd primes and monotone groups for the prime 2,

(ii) $N=G^{\mathfrak{N}}$ (the nilpotent residual of $G$ ) is abelian $($ and $\neq 1)$ and so $G=N H$, $N \cap H=1$ for every Carter subgroup $H$ of $G$,

(iii) $H$ acts on $N$ as power automorphisms and if $\pi$ is the set of primes dividing $N$ then $H_{p}$ is cyclic if $p \in \pi$ and $H_{\pi^{\prime}} / C_{H_{\pi^{\prime}}}(N)$ is cyclic.

(iv) If $q \in \pi^{\prime}, x \notin C_{H_{q}}(N)$ and $y \in C_{H_{q}}(N)$ then $K=\langle x, y\rangle=U\langle x\rangle$ with $U$ cyclic, normal in $K$ and contained in $C_{H}(N)$.

We will need the following result, essentially due to Gaschütz: the version here is due to Lucchini and Tamburini [11]. Let $d(G)$ denote the minimum number of generators of a supersoluble group $G$ and for each isomorphism type of chief factor $A$ (as $G$-module) let $\delta_{G}(A)$ denote the number of complemented chief factors of $G$ isomorphic to $A$ in a fixed chief series and let $\Omega(G)$ denote the set of nonisomorphic complemented chief factors.

Lemma 1.2. With the notation above

$$
d(G)=\max _{A \in \Omega(G)} h_{G}(A)
$$

where $h_{G}(A)=\left(\delta_{G}(A)+1-\theta_{G}(A)\right)$ and $\theta_{G}(A)=1$ if $A$ is a trivial $G$-module, and $\theta_{G}(A)=0$ otherwise.

\section{The proof of Theorem 1}

Suppose first that $G \in \mathcal{M}$.

(i) Since metacyclic groups are supersoluble, we have that $G$ is supersoluble ([6]). We defer the proof that $G$ is metabelian until later. The structure of the Sylow subgroups is covered by the remarks above.

(ii) Since $G$ is supersoluble the nilpotent residual $N$ of $G$ is nilpotent of odd order. Let $p$ be a prime. Suppose that the Sylow $p$-subgroup $M$ of $N$ is non-trivial, and let $K$ be a Hall $p^{\prime}$-subgroup of $G$. We show that all $G$-chief factors of $M / \Phi(M)$ are isomorphic as $K$-modules. Assume this is not true and let $A / B$ be a $G$-chief factor such that $\Phi(M) \leq B<A \leq M$, all $G$-chief factors between $M$ and $A$ are $K$-isomorphic and $A / B$ is not $K$-isomorphic to any $G$-chief factor between $A$ and $M$. Suppose that $A$ is a proper subgroup of $M$. Then $M / B$ is an elementary abelian 
non-cyclic $p$-group and every $G$-chief factor between $B$ and $M$ is non-central in $G$. By [7, Theorem A. 11.5], $M / B$ is a semisimple $K$-module. Let $C / B$ be a $G-$ chief factor below $M$ such that $A \cap C=B$. Write $D=A C$. Since $K / C_{K}(A / B)$ and $K / C_{K}(C / B)$ are both cyclic and $C_{K}(A / B) \cup C_{K}(C / B) \neq K$ we can find an element $k \in K$ such that $C_{K}(A / B)\langle k\rangle=C_{K}(C / B)\langle k\rangle=K$. Then $A / B$ and $C / B$ are non-isomorphic as $\langle k\rangle$-modules. We now have $D\langle k\rangle / B$ a 2-generator group by Lemma 1. Since $(D\langle h\rangle / B)^{\prime}=C / B$ is not cyclic, this group is not metacyclic, a contradiction. Assume now that $M=A$. Then $\Phi(M)$ is a proper subgroup of $B$ and there exists a normal subgroup $Z$ of $G$ such that $B / Z$ and $M / B$ are two non-central chief factors of $G$ which are not $K$-isomorphic. Then $M / Z$ is 2-generated. Arguing as above, there exists an element $h \in K$ such that $C_{K}(M / B)\langle k\rangle=C_{K}(B / Z)\langle h\rangle=$ $K$ and $M\langle h\rangle / Z$ is a 2-generated group which is not metacyclic, a contradiction. Therefore, all $G$-chief factors of $M / \Phi(M)$ are isomorphic as $K$-modules and, by [1, Lemma 2.1.3], $K$ acts as power automorphisms on $M / \Phi(M)$.

Suppose that $M$ is non-abelian. By [13, Lemma 2.3.33], there exist characteristic subgroups $R$ and $S$ of $M$ such that $\Phi(M) \leq S<R$ and $[R, \operatorname{Aut}(M)] \leq S$. In particular, every $G$-chief factor between $\Phi(M)$ and $M$ is central in $G$. This is not possible. Hence $M$ is abelian. It now follows from [5, Lemma 2] (see also [1, Lemma 3.3.39]) that all chief factors of $G$ contained in $M$ are isomorphic as $G$-modules. By [1, Lemma 2.1.3], $p^{\prime}$-elements of $G$ act on $M$ as power automorphisms.

Since $N$ is abelian it is complemented by every Carter subgroup $H$ of $G$ [7, Theorem IV.5.18].

(iii) Let $N_{p}$ and $H_{p}$ be Sylow $p$-subgroups of $N$ and $H$ respectively. If both $N_{p}$ and $H_{p}$ are non-trivial, we show that $H_{p}$ is cyclic and acts on $N_{p}$ as power automorphisms. To see this suppose first that $H_{p}$ is non-cyclic. Since $p$ is odd, $H_{p}$ contains an elementary abelian subgroup $U=\langle u, v\rangle$ of order $p^{2}$. Let $Z=\langle z\rangle \leq N_{p}$ be a minimal normal subgroup of $G$. Since then $Z \leq Z\left(G_{p}\right) \cap N_{p}$ we have $V=$ $\langle u, v, z\rangle$ elementary abelian of order $p^{3}$. Consider a Hall $p^{\prime}$-subgroup $H_{p^{\prime}}$ of $H$. If $h \in H_{p^{\prime}} \backslash C_{H}(Z)$ set $K=\langle h u, v z\rangle$. We claim that $V \leq K$. Since $h$ and $u$ commute, $u$ and $h$ are both powers of $h u$ so that $u, h \in K$. Next $[h, v z]=[h, z]=z^{\alpha}$ and so $z \in K$. This gives $v \in K$ also and so $V \leq K$. We now have $K$ is a 2-generator which is not metacyclic, a contradiction. Thus $H_{p}$ is cyclic. If $G_{p}$ is abelian then $H_{p}$ acts as the trivial power automorphism on $N_{p}$ so we suppose that $G_{p}$ is nonabelian and $H_{p}=\langle u\rangle$. Since $G_{p}=N_{p} H_{p}, N_{p}$ is abelian and $G_{p}$ is a modular group we must have $u \notin Z\left(G_{p}\right)$ and hence acting as a power automorphism on $N_{p}$.

Since we now have $H$ acts on $N_{p}$ as power automorphisms for each prime in $\pi$ it then follows from [4, Theorem 2.3.1] that $H$ acts as power automorphisms on $N$. Since $H_{\pi^{\prime}} / C_{H_{\pi^{\prime}}}\left(N_{p}\right)$ is cyclic for each $p \in \pi$ by [4, Theorem 3.4.1], $H_{\pi^{\prime}} / C_{H_{\pi^{\prime}}}(N)$ is abelian. Since $C_{H_{\pi^{\prime}}}(N)$ is normal in $G$ to show $H_{\pi^{\prime}} / C_{H_{\pi^{\prime}}}(N)$ is cyclic we may assume that $C_{H_{\pi^{\prime}}}(N)=1$. If $H_{\pi^{\prime}}$ is not cyclic it must contain an elementary abelian subgroup $U$ of order $q^{2}$ for some prime $q \in \pi^{\prime}$. It follows from [4, Theorem 3.4.1] that, for any prime $p \in \pi, U / C_{U}\left(N_{p}\right)$ is cyclic and so we can find primes $p_{1}$ and $p_{2}$ in $\pi$ such that $1<C_{U}\left(N_{p_{i}}\right)=V_{i}<U, i=1,2$ 
and $V_{1} V_{2}=U$. If $W_{i}$ is a non-trivial cyclic subgroup of $N_{p_{i}}, i=1,2$, then $T=\left(W_{1} \times W_{2}\right) U \cong\left(W_{1} V_{2}\right) \times\left(W_{2} V_{1}\right)$. Clearly $W_{1} \times W_{2}$ is a maximal abelian subgroup of $T$. Suppose $T=A B$ with $A$ and $B$ cyclic and $A$ normal in $T$. Then $W_{1} \times W_{2} \leq T^{\prime} \leq A$. Since $T / A$ is cyclic, $W_{1} \times W_{2}$ is a proper subgroup of $A$, a contradiction. Thus $H_{\pi^{\prime}} / C_{H_{\pi^{\prime}}}(N)$ is cyclic.

(iv) Let $K=\langle x, y\rangle$ be as in the statement of the theorem. Then $K=C_{K}(N)\langle x\rangle$. If $\langle y\rangle$ is normal in $K$, set $U=\langle y\rangle$ and (iv) is satisfied. Hence suppose that $\langle y\rangle$ is not normal in $K$. Let $M=\langle m\rangle$ be a cyclic subgroup of $N$ chosen so that $C_{K}(M)=$ $C_{K}(N)$. By (iii) $x$ acts as a non-trivial power automorphism on $M$. Since $M K$ is generated by $y m$ and $x, M K$ is metacyclic. Thus $M K=S T$ with $S$ cyclic and normal in $M K$ and $T$ cyclic. If $S=\langle s\rangle$ then we may assume that $s=m u$ with $u \in C_{K}(M)$ and $T \leq K$ since $M \leq(M K)^{\prime} \leq S$. Let $U=\langle u\rangle$. Then $K=U T$. If $T=\langle t\rangle$ then $x=t^{\alpha} u^{\beta}$. If $\alpha$ is a multiple of $q$ then $\langle x, y\rangle<K$, a contradiction. We now have $t^{\alpha}=x u^{-\beta}$ and so $K=U\langle x\rangle$, completing the proof of (iv).

(i) continued. If $G \in \mathcal{M}$, then $G=N H$ is a subdirect product of $G / N$ and $\left(N_{p} H\right) / C_{H}\left(N_{p}\right)$ for each prime $p$ dividing $|N|$. It is an easy deduction from the list of 2-groups in $\mathcal{M}$ to see that these groups are metabelian. Since modular $p$ groups are metabelian, we have $G / N$ metabelian. Since $H / C_{H}\left(N_{p}\right)$ is cyclic, $\left(N_{p} H\right) / C_{H}\left(N_{p}\right)$ is metabelian. Hence $G$ is metabelian.

Suppose now that $G$ satisfies the hypotheses (i)-(iv) and let $X$ be a 2-generator subgroup of $G$. If $X$ is nilpotent then each Sylow subgroup of $X$ is metacyclic and so $X$ is metacyclic. If $X$ is not nilpotent, let $Y=X^{\mathfrak{N}}$. Since $Y \leq N$ and $G$ acts as power automorphisms on $N, X$ acts as power automorphisms on $Y$. If $Y$ is not cyclic then a Sylow $p$-subgroup $Y_{p}$ of $Y$ is not cyclic for some prime $p$ and so as $X$-module $Y_{p} / \Phi\left(Y_{p}\right)$ is the direct product of $j$ isomorphic irreducibles, $j \geq 2$. Now Lemma 1 gives that $X$ has at least $j+1$ generators, a contradiction. Thus $Y$ is cyclic. Let $Z$ be a complement to $Y$ in $X$. Then $Z$ is a 2-generator nilpotent subgroup of $G$ and hence is metacyclic. If $\left(Z_{p}, Y_{p}\right) \neq 1$ then it follows from (iii) that $Z_{p}$ is cyclic. Thus if $\pi$ is the set of primes dividing $|Y|$ we have $Z=S \times T$, where $S$ is a cyclic $\pi$-group and $T$ is a metacyclic $\pi^{\prime}$-group. If $T$ is cyclic, then $Z$ is cyclic and we are finished. If $T$ is not cyclic and $[T, Y]=1$ then $T=U V$ with $U$ cyclic and normal in $T$ (and hence in $Z$ ) and $V$ cyclic. But then $Y U$ is a cyclic normal subgroup of $X$ and $S V$ is cyclic, so that $X=(Y U)(S V)$ is metacyclic, a contradiction.

Thus $T$ is not cyclic and not contained in $C_{Z}(Y)$. From (iii) we have $T / C_{T}(Y)$ cyclic. We can choose generators $x, y$ for $T$ with $x \notin C_{T}(Y)$ and $y \in C_{T}(Y)$. To show this it will be enough to prove it for the Sylow subgroups of $T$. Let $T_{q}$ be the Sylow $q$-subgroup of $T$ and let $a, b$ be generators of $T_{q}$. Since $T_{q} / C_{T_{q}}(Y)$ is cyclic we must have either $a$ or $b$ generates $T_{q}$ modulo $C_{T_{q}}(Y)$; suppose $a$. Then for some integer $\alpha, b a^{\alpha} \in C_{T_{q}}(Y)$. If $x_{q}=a$ and $y_{q}=b a^{\alpha}$ then $T_{q}=\left\langle x_{q}, y_{q}\right\rangle$. Put $x=\prod_{q \in \pi^{\prime}} x_{q}$ and $y=\prod_{q \in \pi^{\prime}}$. Then $T=\langle x, y\rangle$ with $y \in C_{T}(Y)$.

From (iv) we have $T=U V$ with $U \leq C_{T}(Y)$ cyclic and normal in $T$ and $V$ cyclic. Now $U Y$ is cyclic and normal in $X, V S$ is cyclic and $X=(U Y)(V S)$ is metacyclic, a final contradiction. 


\section{Examples}

The first two examples show that if a metacyclic $q$-group acts on a $p$-group as a group of non-trivial power automorphisms the action plays an important role in whether the extension is metacyclic or not.

Let $N=\langle m\rangle$ be cyclic of order 7 and let $H$ be the nonabelian group of order 27 and exponent 9 , generated by $x$ of order 9 and $y$ of order 3 .

Suppose first that $x$ acts trivially on $N$ and $y$ acts non-trivially on $N$ and let $G=N H$. Then $G=S T$ with $S=N \times\langle x\rangle$ and $T=\langle y\rangle$, with $S$ and $T$ cyclic and $S$ normal in $G$. Thus $G$ is metacyclic.

Now suppose that $y$ acts trivially on $N$ and $X$ acts non-trivially on $N$. We then have $C_{H}(N)=\left\langle x^{3}, y\right\rangle$. The unique maximal cyclic normal subgroup of $G=N H$ is $U=\left\langle x^{3}, N\right\rangle$ and $G / U$ is not cyclic. Thus $G$ is not metacyclic.

Since in Theorem 1 the Sylow $q$-subgroups are modular for $q$ odd, it is tempting to conjecture that $x$ acts as a power automorphism on $C_{H}(N)$. The following example shows this need not be true.

Let $X=\langle x\rangle$ be a cyclic group of order 27 and let $Y=\langle y\rangle \leq \operatorname{Aut}(X)$ with $x^{y}=x^{10}$. Let $N=\langle n\rangle$ be a group of order 7 and let $H=X Y$. Define an action of $H$ on $N$ by $n^{y}=n$ and $n^{x}=n^{2}$. Let $G=N H$. We have $C_{H}(N)=\left\langle x^{3}, y\right\rangle$ and $x$ does not act as a power automorphism on $C_{H}(N)$. However $G$ is metacyclic since $U=N\left\langle y x^{3}\right\rangle$ is a cyclic normal subgroup of $G$ and $U X=G$.

\section{References}

[1] A. Ballester-Bolinches, R. Esteban-Romero and M. AsaAd, "Products of Finite Groups", de Gruyter Expositions in Mathematics 53, de Gruyter, Berlin, New York, 2010.

[2] N. BLACKBURN, Generalizations of certain elementary theorems on p-groups, Proc. London Math. Soc. (3) 11, 1961, 1-22.

[3] E. Crestani and F. Menegazzo, On monotone 2-groups, J. Group Theory 15 (2012), 359-383.

[4] C. D. H. Cooper, Power automorphisms of a group, Math. Z. 107 (1968), 335-356.

[5] J. CosSEY and Y. WANG, Finite dinilpotent groups of small derived length, J. Austral. Math. Soc. (A) 67 (1999), 318-328.

[6] K. DOERK, Minimal nicht überauflösbare, endliche Gruppen, Math. Z. 91 (1966), 198-205.

[7] K. Doerk and T. HAWKes, "Finite Soluble Groups", De Gruyter Expositions in Mathematics 4, De Gruyter, Berlin, New York, 1992.

[8] P. FLAVELL, Finite groups in which every two elements generate a soluble subgroup, Invent. Math. 121 (1995), 279-285.

[9] B. HuPPERT, "Endliche Gruppen I", Springer Verlag, Berlin, Heidelberg, New York, 1967.

[10] B. HuPPERT and N. BlACKBURN, "Finite Groups II", Springer Verlag, Berlin, Heidelberg, New York, 1982.

[11] A. LUCCHINI and M. C. TAMBURINI, Minimal generation of finite soluble groups by projectors and normalizers, Glasg. Math. J. 41 (1999), 303-312.

[12] A. MANN, The number of generators of finite p-groups, J. Group Theory 8 (2005), 317-337.

[13] R. SCHMIDT, "Subgroup Lattices of Groups", Walter de Gruyter, Berlin, New York, 1994. 
[14] J. G. Thompson, Nonsolvable finite groups all of whose local subgroups are solvable, Bull. Amer. Math. Soc. 74 (1968), 383-437.

Departament d'Àlgebra

Universitat de València

Dr. Moliner, 50

46100 Burjassot (València), Spain

Adolfo.Ballester@uv.es

Department of Mathematics

Mathematical Sciences Institute

Australian National University

Canberra ACT 2601, Australia

John.Cossey@anu.edu.au 